\title{
Primary ventral hernia: where are we at?
}

\section{G. Campanelli ${ }^{1}$}

Published online: 17 October 2019

(c) Springer-Verlag France SAS, part of Springer Nature 2019

Primary ventral hernia repair continues to be a poorly clarified abdominal hernia treatment.

After all, in the diagnostic phase, merely distinguishing an umbilical hernia from an epigastric one demands in-depth assessment, and the choice of treatment, in particular, needs to be carefully evaluated.

Another extremely important factor that cannot, and must not, be overlooked, is whether the patient presents diastasis of the rectus abdominis muscles. Careful consideration of these three aspects, both in the diagnostic and in the surgical stage, allows us to identify the best solution for the individual case. There are several key points that have already become established, at least with regard to umbilical hernias: first, the importance of size, as this will determine the choice of method (smaller than $1 \mathrm{~cm}$ : simple sutures; larger than $1 \mathrm{~cm}$ : prosthetic repair); second, we know that if prosthetic repair is required, it is undoubtedly preferable to opt for preperitoneal and/or retromuscular placement of the device.

But while some points are already quite clear, most of the choices that need to be made are certainly not. Obviously, an epigastric hernia must be evaluated in the light of the presence or absence of rectus diastasis, and in the not infrequent case of simultaneous umbilical hernia swelling, a correct epigastric repair must undoubtedly protect the entire space between (and behind) the xiphoid process up to the arcuate line (Douglas' line).

But should this surgical choice always be accompanied by correction of the rectus diastasis?

And, if so, which technique should be preferred?

In a patient presenting an uncomplicated umbilical hernia with a diameter of more than $1 \mathrm{~cm}$, no epigastric hernia, and viceversa (i.e. only epigastric hernia without umbilical), but a clear rectus diastasis, what is the correct course of action?

Editor-in-Chief: G. Campanelli.

G. Campanelli

giampiero.campanelli@grupposandonato.it

1 University of Insubria, Gruppo Ospedaliero San Donato, Milan, Italy
Is prosthetic correction of the umbilical or epigastric hernia alone sufficient, or should the prosthesis extend posteriorly to the rectus muscles in the epigastric space as far as the xiphoid process? The extension of mesh positioning is of critical importance. Talking only of overlap is not enough, as reinforcing only one part of the epigastric region makes the rest weak.

Even greater uncertainty surrounds the choice of surgical method: basically, the question is whether to opt for open or laparoscopic (laparoscopic-robotic) surgery. Although, on the one hand, these two methods seem to give entirely comparable results, the choice must be guided by personal experience, and also take into account the patient's physical characteristics. Basically, this means considering not only the site and size of the hernia and the presence, or otherwise, of a diastasis, but also the patient's BMI for example, and the risks associated with it. Hence the laparoscopic approach may be more or less indicated, depending on BMI.

Other much debated questions, both in open and in laparoscopic-robotic surgery concern the issue of where the prosthesis should be positioned: is IPOM still a valuable approach in the era of robotics with the possibility of retromuscular easy positioning of the mesh? And how should such mesh be fixed?

All these points have been addressed and, in part answered, by the authors who have contributed to this issue of HERNIA, which is devoted entirely to the forum on primary ventral hernias.

However, this issue, like previous ones, is also intended to serve as a stimulus and call for further papers dealing with the topic at hand, and above all with the points raised in the discussions.

I therefore feel confident that this issue of HERNIA could prove extremely useful for formulating and subsequently updating guidelines on this topic, too.

Publisher's Note Springer Nature remains neutral with regard to jurisdictional claims in published maps and institutional affiliations. 